九州大学学術情報リポジトリ

Kyushu University Institutional Repository

\title{
Control of vortex chirality in regular polygonal nanomagnets using in-plane magnetic field
}

Yakata, Satoshi

Advanced Electronics Research Division, INAMORI Frontier Research Center, Kyushu University | CREST, Japan Science and Technology Agency

Miyata, Masahiko

Department of Physics, Faculty of Science, Kyushu University | Advanced Electronics Research Division, INAMORI Frontier Research Center, Kyushu University

Nonoguchi, Shogo

Graduate School of Information Science and Electrical Engineering, Kyushu University I

Advanced Electronics Research Division, INAMORI Frontier Research Center, Kyushu University

Wada, Hi rofumi

Department of Physics, Faculty of Science, Kyushu University

他

http://hdl. handle. net/2324/26356

出版情報: Applied Physics Letters. 97 (22)，pp. 222503(1)-222503(3)，2010-11-29. American Institute of Physics

バージョン:

権利関係: (C) 2010 American Institute of Physics 


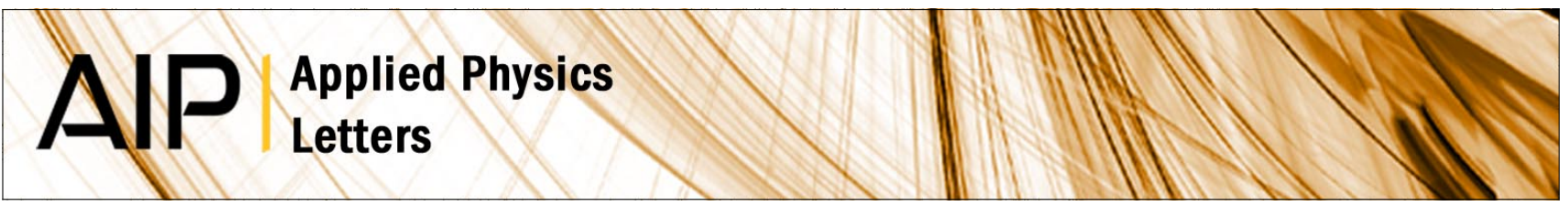

\section{Control of vortex chirality in regular polygonal nanomagnets using in-plane magnetic field}

S. Yakata, M. Miyata, S. Nonoguchi, H. Wada, and T. Kimura

Citation: Appl. Phys. Lett. 97, 222503 (2010); doi: 10.1063/1.3521407

View online: http://dx.doi.org/10.1063/1.3521407

View Table of Contents: http://apl.aip.org/resource/1/APPLAB/v97/i22

Published by the American Institute of Physics.

\section{Related Articles}

Structure, magnetic, and electronic properties of hydrogenated two-dimensional diamond films Appl. Phys. Lett. 102, 073114 (2013)

Thermally activated magnetization switching in a nanostructured synthetic ferrimagnet J. Appl. Phys. 113, 063914 (2013)

Probing nanowire edge roughness using an extended magnetic domain wall Appl. Phys. Lett. 102, 062409 (2013)

Reversal process of a magnetic vortex core under the combined action of a perpendicular field and spin transfer torque

Appl. Phys. Lett. 102, 062401 (2013)

Stripe-vortex transitions in ultrathin magnetic nanostructures

J. Appl. Phys. 113, 054312 (2013)

\section{Additional information on Appl. Phys. Lett.}

Journal Homepage: http://apl.aip.org/

Journal Information: http://apl.aip.org/about/about_the_journal

Top downloads: http://apl.aip.org/features/most_downloaded

Information for Authors: http://apl.aip.org/authors

\section{ADVERTISEMENT}

\section{AIP Applied Physics Letters}

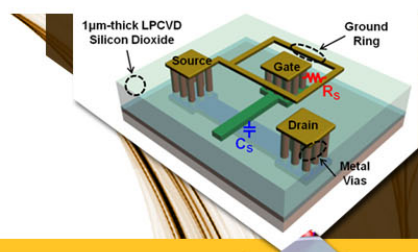

\section{SURFACES AND} INTERFACES

Focusing on physical, chemical, biological structural, optical, magnetic and electrical properties of surfaces and interfaces, and more...

\section{EXPLORE WHAT'S NEW IN APL}

SUBMIT YOUR PAPER NOW!
ENERCY CONVERSION AND STORACE 


\title{
Control of vortex chirality in regular polygonal nanomagnets using in-plane magnetic field
}

\author{
S. Yakata, ${ }^{1,2}$ M. Miyata, ${ }^{1,3}$ S. Nonoguchi, ${ }^{1,4}$ H. Wada, ${ }^{3}$ and T. Kimura ${ }^{1,2, a)}$ \\ ${ }^{1}$ Advanced Electronics Research Division, INAMORI Frontier Research Center, Kyushu University, \\ 744 Motooka, Fukuoka 819-0395, Japan \\ ${ }^{2}$ CREST, Japan Science and Technology Agency, Sanbancho, Tokyo 102-0075, Japan \\ ${ }^{3}$ Department of Physics, Faculty of Science, Kyushu University, Fukuoka 812-8581, Japan \\ ${ }^{4}$ Graduate School of Information Science and Electrical Engineering, Kyushu University, 744 Motooka, \\ Fukuoka 819-0395, Japan
}

(Received 6 October 2010; accepted 2 November 2010; published online 30 November 2010)

\begin{abstract}
The authors have proposed a control method of the magnetic vortex chirality in regular polygonal nanomagnets with an odd number of sides. The asymmetric nucleation energy of the vortex from the uniformly magnetized state enables us to simply control the vortex chirality by an in-plane magnetic field. The reliability of the proposed method has been numerically and experimentally confirmed in the triangle, pentagonal, heptagonal, and nonagonal Permalloy nanomagnets. The authors also confirmed that the vortex chirality is uncontrollable when the number of the side is even. (C) 2010 American Institute of Physics. [doi:10.1063/1.3521407]
\end{abstract}

Patterned magnetic domain structures play an important role for the operations in future spintronic and magnetic storage devices as well as for further understanding of the fundamental spin-related physics. Especially the magnetic vortex structure, which is stabilized in a ferromagnetic circular disk with a diameter less than a micron, has a potential as a unit cell of high density magnetic storage because of negligible magnetostatic interaction and high thermal stability. ${ }^{1,2}$ In addition, the dynamic properties of the magnetic vortex were found to provide various intriguing characteristics in association with the resonant gyration motion of the vortex core. ${ }^{3-6}$ Moreover, it has been demonstrated very recently that nanomagnets with a single magnetic vortex structure destruct cancer cells through the mechanical motions of the nanomagnets. ${ }^{7}$ Therefore, establishing the manipulation method of the magnetic vortex is an important issue not only for further developments of spintronics but also for future biological applications.

A magnetic vortex with a single vortex core can be described by two topological quantities. One is the polarity, which corresponds to the magnetization direction of the vortex core. The polarity strongly correlates to the dynamical gyration motion of the vortex core ${ }^{8,9}$ and the displacement of the vortex core induced by the spin current. ${ }^{10,11}$ The other one is the chirality, which is the rotational direction of the magnetic moment whirling either clockwise $(\mathrm{CW})$ or counterclockwise $(\mathrm{CCW})$. The chirality determines the direction of the vortex shift induced by the in-plane magnetic field. Although the vortex polarity is simply controlled by applying the external perpendicular magnetic field, it is not so easy to control the vortex chirality especially in a circular disk.

In a perfect circular disk, $\mathrm{CW}$ and $\mathrm{CCW}$ states are degenerate because there is no in-plane shape anisotropy. By introducing the asymmetry in the disk, the vortex chirality can be controlled. For example, in the disk with a flat edge on one side, the vortex easily nucleates from the flat edge assisted by the larger demagnetizing field than that at the

${ }^{a)}$ Electronic mail: kimura@ifrc.kyushu-u.ac.jp. round edge when the magnetic field is applied parallel to the flat edge. ${ }^{12,13}$ However, in this method, when the edge of the disk is excessively chipped, the single magnetic vortex structure is not stabilized in the disk. Oppositely, the small chipped region yields the insufficient asymmetry for the chirality control. Thus, the precise adjustment of the chipped area is required for the reliable control of the vortex chirality. Moreover, the residual magnetization becomes nonzero because of the asymmetric shape. Other proposed method using the magnetostatic interaction ${ }^{14}$ or the circular magnetic field ${ }^{15}$ complicates the manipulation of the magnetic vortex.

It is known that the magnetic vortex structure is stabilized also in triangle and square magnetic dots when the lateral dimension is around $1 \mu \mathrm{m} .{ }^{16}$ In the triangle and square nanomagnets, because of the in-plane shape anisotropic energy, we may simply control the vortex chirality by the in-plane magnetic field. Here, we numerically and experimentally investigated the magnetic vortex stabilized in regular polygonal nanomagnets. A simple and reliable control method of the vortex chirality using in-plane magnetic field has been demonstrated.
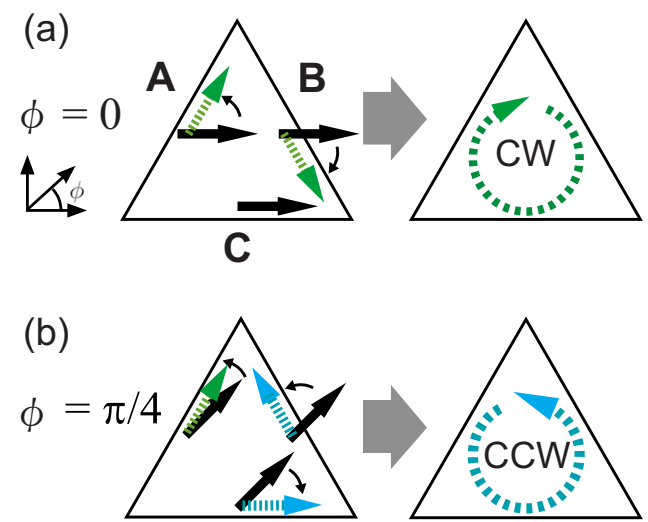

FIG. 1. (Color online) Schematic illustrations of the vortex nucleation processes in a triangle nanomagnet from the uniformly magnetized states with (a) $\phi=0$ and (b) $\phi=\pi / 4$. Solid and dotted arrows indicate the directions of magnetization with magnetic field and without magnetic field, respectively. 
(a) Triangle

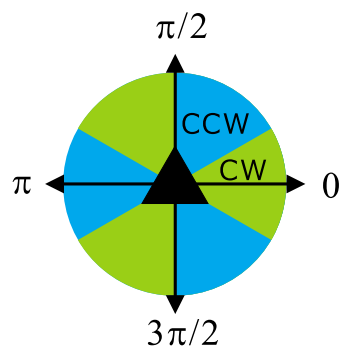

(b) Pentagon

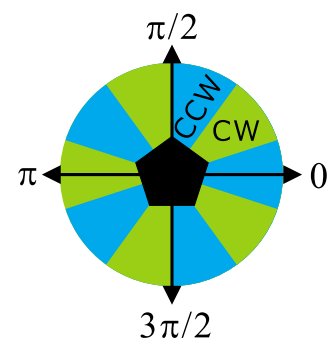

FIG. 2. (Color online) Vortex chiralities formed in (a) triangle and (b) pentagonal nanomagnet as a function of the direction of the initial magnetization obtained by the micromagnetic simulations.

The chirality of the magnetic vortex is strongly related to the nucleation process of the vortex. To form the magnetic vortex from the uniformly magnetized state, the magnetizations at some sides have to be switched. When the number of the side $n$ is odd, the energy of the vortex formation depends on the chirality. For example, as shown in Fig. 1, we consider the formation of a single magnetic vortex from the uniformly magnetized state in a triangle nanomagnet. At $\phi$ $=0$, in order to form the CCW chirality, the magnetizations at the sides $\mathrm{A}$ and $\mathrm{B}$ have to rotate by $2 \pi / 3$ via the highest magnetostatic energy states. Here, the highest magnetization state means that the magnetization of each side is normal to the side. On the other hand, the $\mathrm{CW}$ chirality can be formed by the switching of the magnetizations at the side $\mathrm{C}$ and the $\pi / 6$ rotations of the magnetizations at sides $\mathrm{A}$ and $\mathrm{B}$. This means that the formation of the $\mathrm{CW}$ chirality is easier than that of the CCW chirality. Oppositely, when the magnetization is uniformly saturated at $\phi=\pi / 4$, the formation energy of the vortex with the $\mathrm{CCW}$ chirality is smaller than that with the CW chirality as shown in Fig. 1(b). By using the same manner, the stabilized vortex chirality periodically changes as a function of the direction of the initial magnetization.

To confirm the above scenario, we numerically investigated the remanent states from the various initial directions of the magnetizations using the object-oriented micromagnetic frame network. ${ }^{17}$ Here, we use the Permalloy (Py) parameters with the exchange constant $1.3 \times 10^{-12} \mathrm{~J} / \mathrm{m}$ and the saturation magnetization $1 \mathrm{~T}$. The cell size and the damping parameter are $5 \mathrm{~nm}$ and 0.01 , respectively. The lateral dimension of the regular polygonal magnet has been determined by a circumscribed circle with the diameter of approximately $1 \mu \mathrm{m}$. The thickness of the polygonal nanomagnet is $40 \mathrm{~nm}$. We confirmed that a single magnetic vortex is stabilized in the regular polygonal nanomagnets at the remanent states from the initial state with random magnetizations. Figures 2(a) and 2(b) show the vortex chiralities formed in the triangle and pentagonal nanomagnets, respectively, as a function of the direction of the initial magnetization. The chiralities for the triangle and pentagonal nanomagnet changes periodically at $\pi / 3$ and $\pi / 5$, respectively. These results are consistent with the above expectation. On the other hand, when the number of the side is even, there is no difference in the formation energies between $\mathrm{CW}$ and $\mathrm{CCW}$ states. In fact, in the numerical simulations for square and hexagon nanomagnets, we could not find any manner in the dependence of the vortex chirality on the initialized direction. Moreover, at some angles, the domain structure at the remanent state is not a single vortex. Thus, the present method cannot be applied for the chirality control of the regular polygonal nanomagnet with an even number of sides. We want to emphasize that

\begin{tabular}{|c|c|c|c|}
\hline & Virgin & $\begin{array}{c}\text { Remanent } \\
\text { A }\end{array}$ & $\begin{array}{c}\text { Remanent } \\
\text { B }\end{array}$ \\
\hline $\int_{\text {Triangle }}^{\mathrm{cw}}$ & 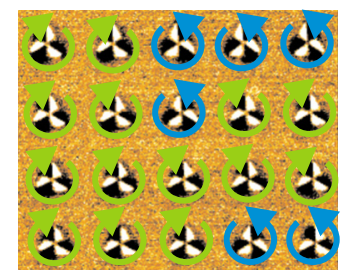 & $\begin{array}{l}A d A d A \\
A d A d A \\
A d A d A d\end{array}$ & 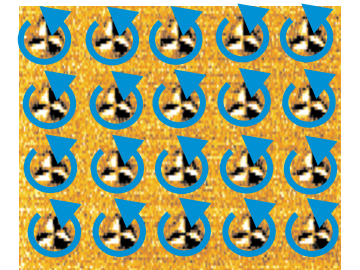 \\
\hline$\underset{\text { Square }}{c w}$ & 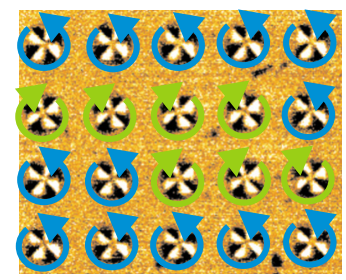 & 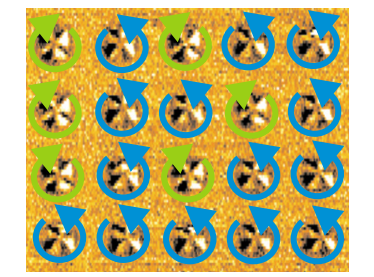 & 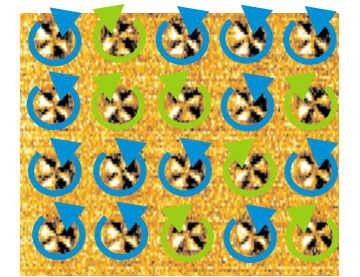 \\
\hline 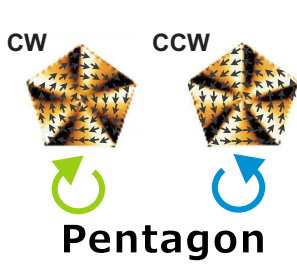 & 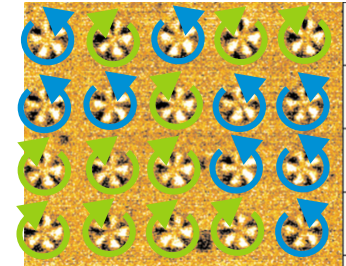 & 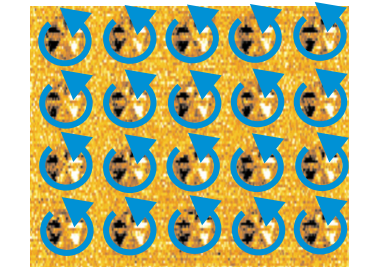 & 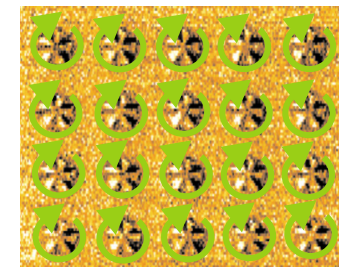 \\
\hline
\end{tabular}

FIG. 3. (Color online) MFM images of virgin state (left) and remanent states after the application of the magnetic field of $80 \mathrm{mT}$ along 0 (center: remanent state A) and $\pi$ (right: remanent state B) in the triangle, square, and pentagonal nanomagnets together with the simulated MFM images obtained from the micromagnetic simulations. 
(a) Heptagon
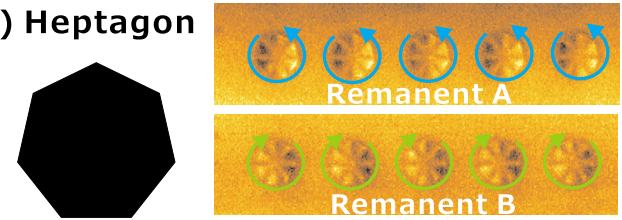

(b) Nonagon
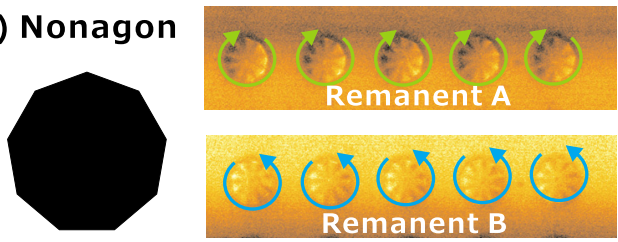

FIG. 4. (Color online) MFM images of (a) heptagonal and (b) nonagonal nanomagnets at remanent state $\mathrm{A}$ initialized by the magnetic field along $\phi$ $=0$ and remanent state $\mathrm{B}$ initialized by the magnetic field along $\phi=\pi$.

these tendencies are reproduced by the calculations in various dimensions of the regular polygonal magnets when a single vortex is stabilized in the polygons.

We then experimentally investigated the magnetic domain structures of regular polygonal Py nanomagnets. The 40-nm-thick Py films were patterned into an array of regular polygons by using electron beam lithography and lift-off technique. The number of sides of polygons was varied from 3 to 9 . The diameter of a circle circumscribed around regular polygonal magnets was $1 \mathrm{um}$. The magnetic domain structures were investigated by using magnetic force microscopy (MFM) (model NanoNavi/E-sweep, SII NanoTechnology, Japan) with low moment probes.

The MFM images in the virgin states for triangle, square, and pentagonal nanomagnets are shown in the left of Fig. 3. Single vortex structures are confirmed in all of the nanomagnets, but their chiralities are randomly distributed. We then apply the external magnetic field of $80 \mathrm{mT}$ at $\phi=0$. As shown in the center of Fig. 3, the chiralities of the triangles and pentagons were confirmed to be fixed in $\mathrm{CW}$ and $\mathrm{CCW}$, respectively. It was also confirmed that the chiralities were changed by changing the direction of the applied magnetic field. As in the right of Fig. 3, the chiralities in the triangle and pentagonal nanomagnets are opposite to those in the center of Fig. 3 after the application of the magnetic field of $80 \mathrm{mT}$ at $\phi=\pi$. On the other hand, the chiralities of the square nanomagnets are randomly distributed even after the application of the in-plane magnetic field. These behaviors are in good agreements with the numerical calculations. Thus, the chirality of regular polygonal nanomagnets can be simply controlled by the application of the in-plane magnetic field when the number of the side is odd.

The validity of the present control method for the magnetic vortex is confirmed also in the heptagonal and nonagonal regular nanomagnets. As in Figs. 4(a) and 4(b), the desired chiralities are reproducible formed. Thus, the vortex chirality in a regular polygonal nanomagnet with an odd number of sides is simply controlled by in-plane magnetic field. However, it may be difficult to apply this method with larger sided polygonal nanomagnets, because the difference in the formation energy between the $\mathrm{CW}$ and $\mathrm{CCW}$ chiralities is smaller than the thermal fluctuation. The use of the ferromagnet with larger saturation magnetization may solve this issue and provide higher thermal stability of the vortex chirality.

In short, we have proposed a simple and reliable control method for the vortex chirality in regular polygonal nanomagnets using in-plane magnetic field. The validity of the method has been confirmed by the micromagnetic simulations and MFM observation. The desired chiralities have been obtained in the triangle, pentagonal heptagonal and nonagonal nanomagnets. By increasing the saturation magnetization, the chirality of the regular polygonal nanomagnets with more sides can be controlled.

One of the authors (T.K.) would like to thank Dr. T. Yamaoka, SII nanotechnology for the technical supports of the MFM observations. This work is partially supported by Industrial Technology Research Grant Program from NEDO and CREST from JST.

${ }^{1}$ R. P. Cowburn, D. K. Koltsov, A. O. Adeyeye, M. E. Welland, and D. M. Tricker, Phys. Rev. Lett. 83, 1042 (1999).

${ }^{2}$ T. Shinjo, T. Okuno, R. Hassdorf, K. Shigeto, and T. Ono, Science $\mathbf{2 8 9}$, 930 (2000).

${ }^{3}$ B. Van Waeyenberge, A. Puzic, H. Stoll, K. W. Chou, T. Tyliszczak, R. Hertel, M. Fähnle, H. Brückl, K. Rott, G. Reiss, I. Neudecker, D. Weiss, C. H. Back, and G. Schütz, Nature (London) 444, 461 (2006).

${ }^{4}$ K. Yamada, S. Kasai, Y. Nakatani, K. Kobayashi, H. Kohno, A. Thiaville, and T. Ono, Nature Mater. 6, 270 (2007).

${ }^{5}$ V. S. Pribiag, I. N. Krivorotov, G. D. Fuchs, P. M. Braganca, O. Ozatay, J. C. Sankey, D. C. Ralph, and R. A. Buhrman, Nat. Phys. 3, 498 (2007).

${ }^{6}$ K. Yamada, S. Kasai, Y. Nakatani, K. Kobayashi, and T. Ono, Appl. Phys. Lett. 93, 152502 (2008).

${ }^{7}$ D.-H. Kim, E. A. Rozhkova, I. V. Ulasov, S. D. Bader, T. Rajh, M. S. Lesniak, and V. Novosad, Nature Mater. 9, 165 (2010).

${ }^{8}$ K. Yu. Guslienko, V. Novosad, Y. Otani, H. Shima, and K. Fukamichi, Phys. Rev. B 65, 024414 (2001).

${ }^{9}$ J. Shibata, K. Shigeto, and Y. Otani, Phys. Rev. B 67, 224404 (2003).

${ }^{10}$ J. Shibata, Y. Nakatani, G. Tatara, H. Kohno, and Y. Otani, Phys. Rev. B 73, 020403(R) (2006).

${ }^{11}$ T. Ishida, T. Kimura, and Y. Otani, Phys. Rev. B 74, 014424 (2006).

${ }^{12}$ M. Schneider, H. Hoffmann, and J. Zweck, Appl. Phys. Lett. 79, 3113 (2001).

${ }^{13}$ T. Kimura Y. Otani, H. Masaki, T. Ishida, R. Antos, and J. Shibata, Appl. Phys. Lett. 90, 132501 (2007).

${ }^{14}$ S. Y. H. Lua, S. S. Kushvaha, Y. H. Wu, K. L. Teo, and T. C. Chong, J. Appl. Phys. 105, 07A319 (2009).

${ }^{15}$ K. Bussmann, G. A. Prinz, S.-F. Cheng, and D. Wang, Appl. Phys. Lett. 75, 2476 (1999).

${ }^{16}$ V. Novosad, M. Grimsditch, J. Darrouzet, J. Pearson, S. D. Bader, V. Metlushko, K. Guslienko, Y. Otani, H. Shima, and K. Fukamichi, Appl. Phys. Lett. 82, 3716 (2003).

${ }^{17}$ See: http://math.nist.gov/oommf/ for micromagnetic simulations. 